\title{
Determinants of early initiation of breastfeeding in Peru: analysis of the 2018 Demographic and Family Health Survey
}

\author{
Akram Hernández-Vásquez ${ }^{1}$, Horacio Chacón-Torrico² \\ 'Universidad San Ignacio de Loyola, Vicerrectorado de Investigación, Centro de Excelencia en Investigaciones Económicas y Sociales en Salud, \\ Lima, Peru; ${ }^{2}$ Facultad de Medicina, Universidad Científica del Sur, Lima, Peru
}

OBJECTIVES: Early initiation of breastfeeding (EIBF) is one of the most cost-effective strategies to reduce neonatal mortality. We sought to determine the prevalence and determinants of EIBF in Peru.

METHODS: We performed a cross-sectional analytical study of the 2018 Peruvian Demographic and Family Health Survey as a secondary data source. In total, 19,595 children born during the 5 years prior to the survey were included in the study. The dependent variable (EIBF status), socio-demographic variables, and pregnancy-related variables were analyzed using a multivariate logistic regression model to identify the determinants of EIBF.

RESULTS: The prevalence of EIBF in the study population was 49.7\%. Cesarean deliveries were associated with a lower likelihood of EIBF (adjusted odds ratio [aOR], 0.06; 95\% confidence interval [CI], 0.05 to 0.07 ) than were vaginal deliveries. Newborns born at public health centers (aOR, 1.37; 95\% CI, 1.15 to 1.65) had a higher rate of EIBF than those not born at public or private health centers. Women from the jungle region (aOR, 2.51; 95\% CI, 2.17 to 2.89) had higher odds of providing EIBF than those from the coast. Mothers with more than a secondary education (aOR, $0.65 ; 95 \% \mathrm{CI}, 0.55$ to 0.76 ) were less likely to breastfeed during the first hour of the newborn's life than women with primary or no education.

CONCLUSIONS: More than half of Peruvian children do not breastfeed during the first hour after birth. The major determinants of EIBF status were the delivery mode and the region of maternal residence. Strategies are needed to promote early breastfeeding practices.

KEY WORDS: Breastfeeding, Newborn, Health surveys, Peru

\section{INTRODUCTION}

Although neonatal mortality (NM) worldwide decreased by half between 1990 and 2017, it is projected that by 2030, 27.8 mil-

\section{Correspondence: Akram Hernández-Vásquez}

Universidad San Ignacio de Loyola, Vicerrectorado de Investigación,

Centro de Excelencia en Investigaciones Económicas y Sociales en

Salud, 550 La Fontana Avenue, Lima 00012, Peru

E-mail: ahernandez@usil.edu.pe

Received: Nov 10, 2019 / Accepted: Dec 25, 2019 / Published: Dec 25, 2019

This article is available from: http://e-epih.org/

(c) This is an open-access article distributed under the terms of the Creative Commons Attribution License (http://creativecommons.org/licenses/by/4.0/), which permits unrestricted use, distribution, and reproduction in any medium, provided the original work is properly cited.

(C) 2019, Korean Society of Epidemiology lion newborns will die during the first month of life [1]. In order to reduce neonatal deaths, the World Health Organization (WHO) recommends a series of essential, cost-effective, and easy-to-implement practices [2]. These practices include hygienic handling of the umbilical cord, thermal control, and early lactation. Evidence based on high-quality data has confirmed the benefits of early lactation for NM [3], and it is estimated that the implementation of large-scale breastfeeding promotion programs could prevent over $11.6 \%$ of newborn deaths and cause a reduction of over 21.9 million disability-adjusted life years [4].

Early initiation of breastfeeding (EIBF) is defined as the intake of breast milk by the newborn within the first hour after birth [5]. The main benefits of EIBF include stimulating colostrum production, reducing postpartum hemorrhage, and promoting exclusive long-term breastfeeding [6,7]. However, it should be noted that 
despite the position of the WHO on the benefits of EIBF, several studies have failed to show a positive relationship between EIBF and exclusive breastfeeding [8,9]. It has also been estimated that up to $22 \%$ of neonatal deaths can be prevented with EIBF [10]. Nonetheless, it has been reported that only around half of newborns worldwide are breastfed within the first hour of life [11]. Likewise, a study of 57 low-income and middle-income countries showed that only $39 \%$ of newborns engaged in EIBF [12].

Several studies have reported that some socio-demographic characteristics, such as place of residence, maternal education level, socioeconomic status, and place of birth, are associated with EIBF $[13,14]$. Cultural beliefs and traditional feeding practices have also been described as important barriers to EIBF [15]. However, making generalizations regarding the determinants of EIBF is difficult due to the presence of considerable regional differences [14]. For countries in Latin America and the Caribbean, there is still little evidence on this issue. Studies conducted in these regions have reported that the prevalence of EIBF among newborns delivered in private-sector facilities was $45.2 \%$, as opposed to $62.8 \%$ among those born at public-sector institutions [12].

In Peru, the 2018 Demographic and Family Health Survey (ENDES, for its acronym in Spanish) reported that over $49.7 \%$ of the children born during the 5 years prior to the survey were breastfed during the first hour after birth. In addition, a multicenter study reported that the prevalence of EIBF in hospitals in Peru was the lowest (17.7\%) of 24 low-income and middle-income countries in Africa, Asia, and Latin America [16]. Peru is a middle- to high-income country, and despite having reached the goal of reducing NM as required by the Millennium Goals for 2015, it still exhibits many inequalities that underlie access to most indicators of child and neonatal health [17]. These differences can be attributed to the geographic and socioeconomic diversity of Peru.

Given the suboptimal rate of EIBF in Peru and the scarce evidence regarding its determinants, this problem must be assessed. For this reason, the aim of the present study was to analyze the socio-demographic and maternal factors related to EIBF in newborns in Peru during the 5 years prior to the 2018 ENDES.

\section{MATERIALS AND METHODS}

\section{Design and population study}

We conducted a secondary analysis of data from the $2018 \mathrm{EN}$ DES. The ENDES was carried out by the Instituto Nacional de Estadística e Informática (INEI) of Peru. ENDES data are freely available and can be obtained from the INEI web portal (http://iinei. inei.gob.pe/microdatos/). The ENDES used a multi-stage stratified random sampling and includes individuals living in the selected households. The details of the methodology of the survey can be consulted in the final technical report [18].

Members of a total of 21,960 households from urban and rural places of the 24 departments of Peru and the province of Callao were interviewed. For the 2018 ENDES, 34,971 women between 15 and 49 years of age and 23,983 children under 5 years old re- siding in the selected households were identified.

\section{Variables and measurements}

According to the WHO, EBIF is defined as breastfeeding within the first hour after birth [5]. In the 2018 ENDES, women were asked about the initiation of breastfeeding for children born in the 5 years prior to the survey. Responses of fewer than 24 hours were recorded in hours. A binary variable was then created and categorized as: (1) EIBF if a child was breastfed immediately or within 1 hour after birth, or (2) non-EIBF if a child was breastfed after 1 hour.

The following independent variables were included in the analysis: age of the woman in years (15-24, 25-34, or 35-49), marital status (never married, separated/divorced/widowed, or married/ cohabiting), level of education (no formal school/primary, secondary, or higher), wealth index quintiles (poorest, poorer, middle, richer, or richest), region of residence (Peru is divided into 3 regions: the coastal region, near the Pacific coastal line [including Lima, the country's capital city]; the highlands region of the Andes; and the jungle, where the Amazon rainforest is located), place of residence (urban or rural), cesarean section (yes or no), place of delivery (private health facility, public health facility, or other), birth order (first, second or third, or fourth or higher), size of the child at birth (small, medium, or large), sex of the newborn (female or male), type of pregnancy (multiple or single), number of prenatal visits (0-3, 4-7, or 8 or more), utilization of breastfeeding training (yes or no), ethnic self-identification (White/mixed-race/ other, Native, or Black/Brown/Zambo), and head of the household (yes or no). The selection and inclusion of these variables was based on an epidemiological criterion and on variables reported in previous ENDES-based studies [19-23].

\section{Statistical analysis}

All analyses were conducted using Stata version 14.2 (Stata Corp., College Station, TX, USA), and the svy command was used to adjust for sampling weights and clustering. Demographic and socioeconomic characteristics, as well as the outcome variable, were described by absolute frequencies and weighted proportions with 95\% confidence intervals [Cis]. The chi-square test was utilized to assess the associations between the explanatory variables and the outcome variable.

Crude odds ratios (ORs) and adjusted odds ratios (aORs) with 95\% CIs were also calculated. A logistic regression model was used to measure the association between the study factors and EIBF status. A bivariate logistic regression (crude analysis) was carried out among the variables of interest. Independent variables with p-values $<0.20$ in the bivariate analysis and factors known from the literature to predict the outcome variable were included in the multiple logistic regression. The OR values with $95 \%$ CIs for both models are presented. Multicollinearity was assessed using the variance inflation factor. A p-value $<0.05$ was considered to indicate statistical significance, and $\mathrm{p}$-values were not corrected for multiple testing. 


\section{Ethics statement}

This study did not require the approval of an ethics committee because it was an analysis of a de-identified secondary dataset of the 2018 ENDES, which is freely and publicly available. The aim of these annually executed surveys under the Demographic and Health Surveys model [24] is to obtain data on a wide range of nationwide development indicators by a governmental agency.

\section{RESULTS}

From the 2018 ENDES population total of 19,696 children, our analysis included 19,595 children born during the 5 years prior to the survey and for whom complete information was available. Table 1 shows the distribution of the study population according to the variables studied. Approximately 9 out of 10 deliveries (92.7\%) occurred in a private or public health care facility. The mean maternal age at the time of the survey was $30.4 \pm 7.1$ years. The most common marital status was married/cohabiting (84.5\%), and the most common places and regions of residence were urban places and the coast region, respectively, with $75.0 \%$ of participants residing in an urban place and $56.0 \%$ from the coastal region.

Approximately half of the women (49.7\%) surveyed reported having breastfed their newborn during the first hour after birth. This proportion varied significantly based on the characteristics evaluated, with the exceptions of marital status and whether the woman was the head of the household (Table 2). With regard to wealth index, immediate breastfeeding ranged from $70.6 \%$ in the lowest wealth quintile to $27.5 \%$ in the highest quintile. Likewise, there was a major difference in the use of EIBF according to delivery mode, with a higher prevalence of EIBF following vaginal delivery $(71.1 \%)$ than cesarean section. EIBF also varied greatly by education level, with EIBF being engaged in by $68.7 \%$ of women with primary or no education and by $36.3 \%$ of women with more than a secondary education.

Table 3 shows the raw and adjusted binary logistic regression models between the independent variables and EIBF status. Women who experienced a cesarean delivery were $94 \%$ less likely to breastfeed during the first hour after delivery than women who experienced a vaginal birth, thereby showing delivery mode to be the strongest determinant in both the crude and adjusted models (OR, 0.05; aOR, 0.06). Age group was not associated with frequency of immediate breastfeeding in either the crude or aOR model. The socio-demographic variable showing the strongest association with EIBF was the region of maternal residence. As such, women from the jungle region were more likely to breastfeed during the first hour than those from the coast (aOR, 2.51; 95\% CI, 2.17 to 2.89 ). In contrast, women who ethnically identified themselves as "Native" were $14 \%$ less likely to breastfeed immediately than women who self-identified as "White/mixed-race/other" (aOR, 0.86 ; $95 \%$ CI, 0.76 to 0.97 ).

With regard to the pregnancy and childbirth variables, women who experienced a single birth were more likely (aOR, 2.81; 95\% CI, 1.35 to 5.85 ) to engage in EIBF than those who experienced
Table 1. Characteristics of the women and children included in the study $(n=19,595)$

\begin{tabular}{|c|c|c|}
\hline Characteristics & $\mathrm{n}$ & Weighted $\%^{1}$ \\
\hline \multicolumn{3}{|l|}{ Early initiation of breastfeeding } \\
\hline No & 9,088 & 50.3 \\
\hline Yes & 10,507 & 49.7 \\
\hline \multicolumn{3}{|l|}{ Age (yr) } \\
\hline $15-24$ & 4,710 & 22.9 \\
\hline $25-34$ & 9,037 & 46.5 \\
\hline $35-49$ & 5,848 & 30.6 \\
\hline \multicolumn{3}{|l|}{ Marital status } \\
\hline Never married & 1,028 & 5.1 \\
\hline Separated/divorced/widowed & 2,033 & 10.4 \\
\hline Married/cohabiting & 16,534 & 84.5 \\
\hline \multicolumn{3}{|l|}{ Level of education } \\
\hline No formal school/primary & 4,163 & 19.5 \\
\hline Secondary & 8,856 & 44.2 \\
\hline Higher & 6,576 & 36.3 \\
\hline \multicolumn{3}{|l|}{ Wealth Index } \\
\hline Poorest & 5,519 & 23.6 \\
\hline Poorer & 5,110 & 23.1 \\
\hline Middle & 3,874 & 20.1 \\
\hline Richer & 2,937 & 17.5 \\
\hline Richest & 2,155 & 15.7 \\
\hline \multicolumn{3}{|l|}{ Region of residence } \\
\hline Coast & 8,373 & 56.0 \\
\hline Andean & 6,598 & 27.4 \\
\hline Jungle & 4,624 & 16.6 \\
\hline \multicolumn{3}{|l|}{ Place of residence } \\
\hline Rural & 5,830 & 25.0 \\
\hline Urban & 13,765 & 75.0 \\
\hline \multicolumn{3}{|l|}{ Mode of delivery } \\
\hline Vaginal delivery & 13,271 & 64.5 \\
\hline Cesarean delivery & 6,324 & 35.5 \\
\hline \multicolumn{3}{|l|}{ Place of delivery } \\
\hline Other ${ }^{2}$ & 1,347 & 7.3 \\
\hline Private health center & 1,820 & 14.5 \\
\hline Public health center & 16,428 & 78.2 \\
\hline \multicolumn{3}{|l|}{ Order of birth } \\
\hline 1 & 6,156 & 32.9 \\
\hline $2-3$ & 9,824 & 50.3 \\
\hline$\geq 4$ & 3,615 & 16.8 \\
\hline \multicolumn{3}{|l|}{ Newborn size } \\
\hline Small & 4,163 & 20.9 \\
\hline Medium & 10,232 & 51.5 \\
\hline Large & 5,200 & 27.6 \\
\hline \multicolumn{3}{|l|}{ Newborn sex } \\
\hline Female & 9,577 & 48.5 \\
\hline Male & 10,018 & 51.5 \\
\hline \multicolumn{3}{|l|}{ Type of pregnancy } \\
\hline Multiple & 186 & 1.1 \\
\hline Single & 19,409 & 98.9 \\
\hline
\end{tabular}


Table 1. Continued

\begin{tabular}{lrc}
\hline Characteristics & $\mathrm{n}$ & Weighted ${ }^{1}$ \\
\hline Antenatal controls & & \\
$0-3$ & 764 & 3.5 \\
$4-7$ & 4,737 & 23.4 \\
$\geq 8$ & 14,094 & 73.1 \\
Breastfeeding training & & \\
$\quad$ No & 5,804 & 30.2 \\
Yes & 13,791 & 69.8 \\
Ethnic self-identification & & \\
White/mixed-race/others & 11,136 & 63.0 \\
Native & 6,651 & 26.7 \\
Black/Brown/"Zambo" & 1,808 & 10.3 \\
Head of the household & & \\
No & 17,081 & 88.1 \\
Yes & 2,514 & 11.9 \\
\hline
\end{tabular}

'Estimates include the weights and Demographic and Family Health Survey sample specifications.

${ }^{2}$ Neither private nor public health centers.

Table 2. Factors associated with early initiation of breastfeeding

\begin{tabular}{|c|c|c|c|}
\hline \multirow{2}{*}{ Characteristics } & \multicolumn{2}{|c|}{ Breastfeeding, $\%^{1}$} & \multirow{2}{*}{$p$-value } \\
\hline & No & Yes & \\
\hline \multicolumn{4}{|l|}{ Age (yr) } \\
\hline $15-24$ & 43.8 & 56.2 & $<0.001$ \\
\hline $25-34$ & 50.0 & 50.0 & \\
\hline $35-49$ & 55.3 & 44.7 & \\
\hline Marital status & & & 0.407 \\
\hline Never married & 50.9 & 49.1 & \\
\hline Separated/divorced/widowed & 51.9 & 48.1 & \\
\hline Married/cohabiting & 50.0 & 50.0 & \\
\hline Level of education & & & $<0.001$ \\
\hline No formal school/primary & 31.3 & 68.7 & \\
\hline Secondary & 47.5 & 52.5 & \\
\hline Higher & 63.7 & 36.3 & \\
\hline Wealth Index & & & $<0.001$ \\
\hline Poorest & 29.4 & 70.6 & \\
\hline Poorer & 43.8 & 56.2 & \\
\hline Middle & 54.5 & 45.5 & \\
\hline Richer & 61.7 & 38.3 & \\
\hline Richest & 72.5 & 27.5 & \\
\hline Region of residence & & & $<0.001$ \\
\hline Coast & 60.8 & 39.2 & \\
\hline Andean & 40.2 & 59.8 & \\
\hline Jungle & 30.9 & 69.1 & \\
\hline Place of residence & & & $<0.001$ \\
\hline Rural & 31.5 & 68.5 & \\
\hline Urban & 56.4 & 43.6 & \\
\hline Mode of delivery & & & $<0.001$ \\
\hline Vaginal delivery & 28.9 & 71.1 & \\
\hline Cesarean delivery & 89.0 & 11.0 & \\
\hline
\end{tabular}

Table 2. Continued

\begin{tabular}{|c|c|c|c|}
\hline \multirow{2}{*}{ Characteristics } & \multicolumn{2}{|c|}{ Breastfeeding, $\%^{1}$} & \multirow{2}{*}{$\mathrm{p}$-value } \\
\hline & No & Yes & \\
\hline Place of delivery & & & $<0.001$ \\
\hline Other $^{3}$ & 27.2 & 72.8 & \\
\hline Private health center & 79.1 & 20.9 & \\
\hline Public health center & 47.0 & 53.0 & \\
\hline Order of birth & & & $<0.001$ \\
\hline 1 & 55.7 & 44.3 & \\
\hline $2-3$ & 50.6 & 49.4 & \\
\hline$\geq 4$ & 38.2 & 61.8 & \\
\hline Newborn size & & & $<0.001$ \\
\hline Small & 53.3 & 46.7 & \\
\hline Medium & 47.0 & 53.0 & \\
\hline Large & 53.8 & 46.2 & \\
\hline Newborn sex & & & 0.013 \\
\hline Female & 49.0 & 51.0 & \\
\hline Male & 51.4 & 48.6 & \\
\hline Type of pregnancy & & & $<0.001$ \\
\hline Multiple & 88.2 & 11.8 & \\
\hline Single & 49.8 & 50.2 & \\
\hline Antenatal controls & & & $<0.001$ \\
\hline $0-3$ & 41.8 & 58.2 & \\
\hline $4-7$ & 48.1 & 51.9 & \\
\hline$\geq 8$ & 51.3 & 48.7 & \\
\hline Breastfeeding training & & & 0.007 \\
\hline No & 52.3 & 47.7 & \\
\hline Yes & 49.3 & 50.7 & \\
\hline Ethnic self-identification & & & $<0.001$ \\
\hline White/mixed-race/others & 53.6 & 46.4 & \\
\hline Native & 44.1 & 55.9 & \\
\hline Black/Brown/"Zambo" & 45.2 & 54.8 & \\
\hline Head of the household & & & 0.865 \\
\hline No & 50.2 & 49.8 & \\
\hline Yes & 50.4 & 49.6 & \\
\hline
\end{tabular}

Values are presented as weighted \% of the row unless otherwise indicated.

'Estimates include the weights and Demographic and Family Health Survey sample specifications.

2Using chi-square test statistics.

${ }^{3}$ Neither private nor public health centers.

multiple births. In addition, the size of the newborn was also moderately associated with a higher probability of EIBF in the adjusted model, with aOR values of 1.37 (95\% CI, 1.21 to 1.54 ) and 1.20 ( $95 \%$ CI, 1.04 to 1.39 ) for medium and large infants, respectively, compared to small infants. Finally, birth order which is related to the woman's parity, was also associated with a higher probability of EIBF, with women with 4 or more deliveries being $21 \%$ more likely to perform EIBF than those delivering their first child (aOR, $1.21 ; 95 \%$ CI, 1.01 to 1.45 ). 
Table 3. Crude and adjusted ORs of early breastfeeding for several socioeconomic, pregnancy, and birth variables

\begin{tabular}{|c|c|c|c|c|}
\hline Variables & OR $(95 \% \mathrm{Cl})^{1}$ & $\mathrm{p}$-value & $\mathrm{aOR}(95 \% \mathrm{Cl})^{1,2}$ & $\mathrm{p}$-value \\
\hline \multicolumn{5}{|l|}{ Age (yr) } \\
\hline $15-24$ & 1.00 (reference) & - & 1.00 (reference) & - \\
\hline $25-34$ & $0.78(0.71,0.85)$ & $<0.001$ & $0.94(0.83,1.07)$ & 0.378 \\
\hline $35-49$ & $0.63(0.57,0.70)$ & $<0.001$ & $0.89(0.76,1.05)$ & 0.159 \\
\hline Marital status & & & Not included & \\
\hline Never married & 1.00 (reference) & - & - & - \\
\hline Separated/divorced/widowed & $0.96(0.79,1.16)$ & 0.667 & - & - \\
\hline Married/cohabiting & $1.04(0.89,1.21)$ & 0.649 & - & - \\
\hline \multicolumn{5}{|l|}{ Level of education } \\
\hline No formal school/primary & 1.00 (reference) & - & 1.00 (reference) & - \\
\hline Secondary & $0.50(0.45,0.56)$ & $<0.001$ & $0.72(0.64,0.82)$ & $<0.001$ \\
\hline Higher & $0.26(0.23,0.29)$ & $<0.001$ & $0.65(0.55,0.76)$ & $<0.001$ \\
\hline \multicolumn{5}{|l|}{ Wealth Index } \\
\hline Poorest & 1.00 (reference) & - & 1.00 (reference) & - \\
\hline Poorer & $0.53(0.48,0.60)$ & $<0.001$ & $0.94(0.81,1.10)$ & 0.430 \\
\hline Middle & $0.35(0.31,0.39)$ & $<0.001$ & $0.84(0.70,1.00)$ & 0.055 \\
\hline Richer & $0.26(0.23,0.29)$ & $<0.001$ & $0.82(0.67,1.01)$ & 0.057 \\
\hline Richest & $0.16(0.14,0.18)$ & $<0.001$ & $0.77(0.61,0.98)$ & 0.033 \\
\hline \multicolumn{5}{|l|}{ Region of residence } \\
\hline Coast & 1.00 (reference) & - & 1.00 (reference) & - \\
\hline Andean & $2.31(2.11,2.53)$ & $<0.001$ & $1.49(1.30,1.70)$ & $<0.001$ \\
\hline Jungle & $3.47(3.12,3.86)$ & $<0.001$ & $2.51(2.17,2.89)$ & $<0.001$ \\
\hline \multicolumn{5}{|l|}{ Place of residence } \\
\hline Rural & 1.00 (reference) & - & 1.00 (reference) & - \\
\hline Urban & $0.35(0.32,0.39)$ & $<0.001$ & $0.90(0.78,1.05)$ & 0.167 \\
\hline \multicolumn{5}{|l|}{ Mode of delivery } \\
\hline Vaginal delivery & 1.00 (reference) & - & 1.00 (reference) & - \\
\hline Cesarean delivery & $0.05(0.04,0.06)$ & $<0.001$ & $0.06(0.05,0.07)$ & $<0.001$ \\
\hline \multicolumn{5}{|l|}{ Place of delivery } \\
\hline Other ${ }^{3}$ & 1.00 (reference) & - & 1.00 (reference) & - \\
\hline Private health center & $0.10(0.08,0.12)$ & $<0.001$ & $1.18(0.90,1.54)$ & 0.225 \\
\hline Public health center & $0.42(0.36,0.50)$ & $<0.001$ & $1.37(1.15,1.65)$ & 0.001 \\
\hline \multicolumn{5}{|l|}{ Order of birth } \\
\hline 1 & 1.00 (reference) & - & 1.00 (reference) & - \\
\hline $2-3$ & $1.22(1.13,1.33)$ & $<0.001$ & $1.07(0.94,1.21)$ & 0.293 \\
\hline$\geq 4$ & $2.03(1.82,2.27)$ & $<0.001$ & $1.21(1.01,1.45)$ & 0.040 \\
\hline \multicolumn{5}{|l|}{ Newborn size } \\
\hline Small & 1.00 (reference) & - & 1.00 (reference) & - \\
\hline Medium & $1.29(1.17,1.42)$ & $<0.001$ & $1.37(1.21,1.54)$ & $<0.001$ \\
\hline Large & $0.98(0.88,1.09)$ & 0.736 & $1.20(1.04,1.39)$ & 0.011 \\
\hline \multicolumn{5}{|l|}{ Newborn sex } \\
\hline Female & 1.00 (reference) & - & 1.00 (reference) & - \\
\hline Male & $0.91(0.84,0.98)$ & 0.013 & $0.93(0.85,1.03)$ & 0.158 \\
\hline \multicolumn{5}{|l|}{ Type of pregnancy } \\
\hline Multiple & 1.00 (reference) & - & 1.00 (reference) & - \\
\hline Single & $7.55(4.43,12.87)$ & $<0.001$ & $2.81(1.35,5.85)$ & 0.006 \\
\hline \multicolumn{5}{|l|}{ Antenatal controls } \\
\hline $0-3$ & 1.00 (reference) & - & 1.00 (reference) & - \\
\hline $4-7$ & $0.77(0.63,0.95)$ & 0.013 & $1.03(0.80,1.31)$ & 0.833 \\
\hline$\geq 8$ & $0.68(0.56,0.83)$ & $<0.001$ & $1.20(0.94,1.53)$ & 0.136 \\
\hline
\end{tabular}


Table 3. Continued

\begin{tabular}{|c|c|c|c|c|}
\hline Variables & OR $(95 \% \mathrm{Cl})^{1}$ & $p$-value & $\mathrm{aOR}(95 \% \mathrm{Cl})^{1,2}$ & $\mathrm{p}$-value \\
\hline \multicolumn{5}{|l|}{ Breastfeeding training } \\
\hline No & 1.00 (reference) & - & 1.00 (reference) & - \\
\hline Yes & $1.13(1.03,1.23)$ & 0.007 & $0.93(0.83,1.04)$ & 0.228 \\
\hline \multicolumn{5}{|l|}{ Ethnic self-identification } \\
\hline White/mixed-race/others & 1.00 (reference) & - & 1.00 (reference) & - \\
\hline Native & $1.47(1.35,1.59)$ & $<0.001$ & $0.86(0.76,0.97)$ & 0.012 \\
\hline Black/Brown/"Zambo" & $1.40(1.23,1.59)$ & $<0.001$ & $1.02(0.87,1.20)$ & 0.808 \\
\hline Head of the household & & & Not included & \\
\hline No & 1.00 (reference) & - & - & - \\
\hline Yes & $0.99(0.88,1.11)$ & 0.865 & - & - \\
\hline
\end{tabular}

$\mathrm{OR}$, odds ratio; $\mathrm{Cl}$, confidence interval; aOR, adjusted odds ratio.

'Estimates include the weights and Demographic and Family Health Survey sample specifications.

${ }^{2}$ Adjusted by all the variables shown in the column that obtained a p-value less than 0.2 in the crude analysis.

${ }^{3}$ Neither private nor public health centers.

\section{DISCUSSION}

The main objective of this study was to identify the determinants of EIBF among 15-year-old to 49-year-old women in Peru using the 2018 ENDES as the data source. According to this dataset, approximately half (49.7\%) of newborns were breastfed during the first hour after birth. While this constitutes a slight increase in EIBF compared to the previous year (48.2\%), a constant decline has been observed since 2013, when the frequency of EIBF peaked at $55.6 \%$ [18]. The results of the present study show that the type of pregnancy and maternal region of residence had the greatest association with EIBF

Multiple studies have reported the mode of delivery to be one of the major determinants of EIBF status in newborns [20,25], with delivery by cesarean section being associated with non-compliance with immediate breastfeeding. This route of delivery induces barriers, such as a delay in skin-to-skin contact between mother and child due to anesthesia, as well as the fatigue associated with a prolonged birth $[26,27]$. Taking into account the progressive increase in cesarean sections both globally and locally, knowledge of the negative effects of this procedure on immediate breastfeeding is important to prevent neonatal deaths.

In contrast, a lower prevalence of EIBF was observed among mothers with a higher level of education. This contrasts with the findings of studies conducted in India [19], Ethiopia [20], and Nigeria [28], which have described that the higher the education level of the mother, the higher the likelihood of EIBF. As shown in a study performed by Islam et al. [29] in Bangladesh, it is thought that other contextual factors can shape the relationship between EIBF and maternal education level. Highly educated women also have a high rate of cesarean sections, which could explain this inverse relationship in the Peruvian population. Despite these results, we believe that education is an important factor associated with higher EIBF rates and that it should be taken into consideration in public health care approaches aimed at promoting new- born health.

Additionally, we found that newborns of mothers from a greater wealth quintile had a lower probability of early breastfeeding. This contrasts with the results of several studies in which a higher socioeconomic level was a positive determinant of EIBF $[20,26]$. It is possible that the association found in this study was determined by practices conducted by the Peruvian population. Another possible explanation is that greater purchasing power allows wealthier mothers to acquire pre-milk supplementation for newborns.

In contrast with the results of previous studies [19], urban or rural place of residence was not a determinant of EIBF status. However, it was observed that newborns of women originating from the jungle region were much more likely to receive immediate breastfeeding than those of women from the coast. It is thought that other cultural and social determinants related to geographical location influence EIBF. These determinants may include low exposure to pro-formula feeding propaganda and the low socioeconomic status associated with the jungle region of residence $[30,31]$.

Several studies have described how access to health services, mainly prenatal care, is a determinant of EIBF [19,32]. However, in our study, this association was not significant. Possible explanations may be poor breastfeeding counseling during antenatal visits that fail to promote this practice, the fragmentation of the Peruvian health system, or the lack of national strategies for the promotion of immediate breastfeeding. It has been reported that the promotion of breastfeeding in health centers in Peru is influenced by the over-demand for health services, poor staff training, and the influence of the formula industry [33]. It is imperative to structure health programs that provide information to pregnant women about the benefits of EIBF. In light of the information collected, the efforts made at promoting EIBF at the primary care level are not effective enough.

Birth order of 3 or more previous deliveries was moderately as- 
sociated with a higher prevalence of EIBF. Similar results were also found in Malawi; in that study, birth order of at least 1 previous delivery was associated with an aOR of 1.30 (95\% CI, 1.06 to 1.67) compared to nulliparous women [34]. It is known that nulliparous women generally have little to no knowledge of pregnancy and childbirth, which could be an important factor influencing attitudes and practices during the first hour after birth [19]. According to the results obtained in the present study, nulliparous women should be prioritized as recipients of breastfeeding counseling and immediate newborn care practices.

Similarly, deliveries at health centers were associated with a higher prevalence of EIBF, especially those at private centers. While previous studies have described lower rates of EIBF associated with deliveries at health centers in Bangladesh [28], a positive association with EIBF has alternatively been reported [34]. Indeed, childbirth care by qualified personnel is designed to encourage pregnant women to start breastfeeding. We found a stronger association with EIBF at private health centers, which likely provide higher-quality training and reinforcement of breastfeeding counseling.

The design of this study was cross-sectional, which was one of the major limitations given its incapacity for establishing causal associations. In addition, when using a secondary data sources, not all potential confounding factors, such as cultural practices or access to lacteal supplementation products, were monitored. An additional bias is that of memory, since the information collected was based entirely on the ability of the women to remember the time at which breastfeeding was initiated. Despite these limitations, the ENDES uses standardized procedures and is performed by trained examiners, which, along with its complex sample design, guarantee the quality, adequate measurement, and national representativeness of the information.

This study evaluated the demographic and health factors associated with EIBF in a representative sample of Peruvian women aged 15 to 49 years. Factors such as mode of delivery, education level, region of residence, place of delivery, birth order, type of pregnancy, and ethnic self-identification were associated with EIBF status. Knowledge of these specific factors in both Peru and countries with similar characteristics in Latin America and the Caribbean may be useful for the design, planning, and execution of breastfeeding promotion strategies aimed at directly and permanently affecting neonatal health.

\section{CONFLICT OF INTEREST}

The authors have no conflicts of interest to declare for this study.

\section{ACKNOWLEDGEMENTS}

The authors would like to thank the National Institute of Statistics and Informatics of Peru for making the database available.

\section{AUTHOR CONTRIBUTIONS}

Conceptualization: AHV. Data curation: AHV. Formal analysis: AHV. Funding acquisition: None. Methodology: AHV, HCT. Project administration: HCT. Visualization: AHV, HCT. Writing original draft: HCT. Writing - review \& editing: AHV, HCT.

\section{ORCID}

Akram Hernández-Vásquez: https://orcid.org/0000-0003-14312526; Horacio Chacón-Torrico: https://orcid.org/0000-0003-45732099

\section{REFERENCES}

1. Hug L, Alexander M, You D, Alkema L; UN Inter-agency Group for Child Mortality Estimation. National, regional, and global levels and trends in neonatal mortality between 1990 and 2017, with scenario-based projections to 2030: a systematic analysis. Lancet Glob Health 2019;7:e710-e720.

2. Lassi ZS, Haider BA, Bhutta ZA. Community-based intervention packages for reducing maternal and neonatal morbidity and mortality and improving neonatal outcomes. Cochrane Database Syst Rev 2010:CD007754.

3. NEOVITA Study Group. Timing of initiation, patterns of breastfeeding, and infant survival: prospective analysis of pooled data from three randomised trials. Lancet Glob Health 2016;4:e266e275.

4. Bhutta ZA, Ahmed T, Black RE, Cousens S, Dewey K, Giugliani E, et al. What works? Interventions for maternal and child undernutrition and survival. Lancet 2008;371:417-440.

5. World Health Organization. Global strategy for infant and young child feeding: the optimal duration of exclusive breastfeeding; 2001 [cited 2019 Dec 26]. Available from: https://apps.who.int/ iris/handle/10665/78801.

6. Begum K, Dewey KG. Impact of early initiation of exclusive breastfeeding on newborn deaths; 2010 [cited 2019 Nov 1]. Available from: https://breastcrawl.org/pdf/impact-of-early-initiation.pdf.

7. World Health Organization. Protecting, promoting and supporting breastfeeding in facilities providing maternity and newborn services: guideline; 2017 [cited 2019 Nov 1]. Available from: https://www.who.int/nutrition/publications/guidelines/breastfeeding-facilities-maternity-newborn/en/.

8. Ferreira HL, Oliveira MF, Bernardo EB, Almeida PC, Aquino PS, Pinheiro AK. Factors associated with adherence to the exclusive breastfeeding. Cien Saude Colet 2018;23:683-690.

9. Finnie S, Peréz-Escamilla R, Buccini G. Determinants of early breastfeeding initiation and exclusive breastfeeding in Colombia. Public Health Nutr 2019:1-10.

10. Edmond KM, Zandoh C, Quigley MA, Amenga-Etego S, Owusu-Agyei S, Kirkwood BR. Delayed breastfeeding initiation increases risk of neonatal mortality. Pediatrics 2006;117:e380-e386.

11. Victora CG, Requejo JH, Barros AJ, Berman P, Bhutta Z, Boerma 
T, et al. Countdown to 2015: a decade of tracking progress for maternal, newborn, and child survival. Lancet 2016;387:20492059.

12. Oakley L, Benova L, Macleod D, Lynch CA, Campbell OM. Early breastfeeding practices: descriptive analysis of recent Demographic and Health Surveys. Matern Child Nutr 2018;14:e12535.

13. Mukora-Mutseyekwa F, Gunguwo H, Mandigo RG, Mundagowa P. Predictors of early initiation of breastfeeding among Zimbabwean women: secondary analysis of ZDHS 2015. Matern Health Neonatol Perinatol 2019;5:2.

14. Ekubay M, Berhe A, Yisma E. Initiation of breastfeeding within one hour of birth among mothers with infants younger than or equal to 6 months of age attending public health institutions in Addis Ababa, Ethiopia. Int Breastfeed J 2018;13:4.

15. Sharma IK, Byrne A. Early initiation of breastfeeding: a systematic literature review of factors and barriers in South Asia. Int Breastfeed J 2016;11:17.

16. Takahashi K, Ganchimeg T, Ota E, Vogel JP, Souza JP, Laopaiboon $\mathrm{M}$, et al. Prevalence of early initiation of breastfeeding and determinants of delayed initiation of breastfeeding: secondary analysis of the WHO Global Survey. Sci Rep 2017;7:44868.

17. Huicho L, Segura ER, Huayanay-Espinoza CA, de Guzman JN, Restrepo-Méndez MC, Tam Y, et al. Child health and nutrition in Peru within an antipoverty political agenda: a Countdown to 2015 country case study. Lancet Glob Health 2016;4:e414-e426.

18. Instituto Peruano de Estadística e Informática. Demographic and Family Health Survey 2018 [cited 2019 Nov 1]. Available from: https://www.inei.gob.pe/media/MenuRecursivo/publicaciones_ digitales/Est/Lib1656/index1.html (Spanish).

19. Senanayake P, O'Connor E, Ogbo FA. National and rural-urban prevalence and determinants of early initiation of breastfeeding in India. BMC Public Health 2019;19:896.

20. John JR, Mistry SK, Kebede G, Manohar N, Arora A. Determinants of early initiation of breastfeeding in Ethiopia: a populationbased study using the 2016 demographic and health survey data. BMC Pregnancy Childbirth 2019;19:69.

21. Adewuyi EO, Zhao Y, Khanal V, Auta A, Bulndi LB. Rural-urban differences on the rates and factors associated with early initiation of breastfeeding in Nigeria: further analysis of the Nigeria demographic and health survey, 2013. Int Breastfeed J 2017;12:51.

22. Hernández-Vásquez A, Vargas-Fernández R, Bendezu-Quispe G. Factors associated with the quality of prenatal care in Peru. Rev Peru Med Exp Salud Publica 2019;36:178-187 (Spanish).

23. Matanda DJ, Mittelmark MB, Urke HB, Amugsi DA. Reliability of demographic and socioeconomic variables in predicting early initiation of breastfeeding: a replication analysis using the Kenya Demographic and Health Survey data. BMJ Open 2014;4:e005194.

24. Demographic and Health Surveys (DHS). DHS overview [cited 2018 Oct 11]. Available from: https://dhsprogram.com/WhatWe-Do/Survey-Types/DHS.cfm

25. Patel A, Bucher S, Pusdekar Y, Esamai F, Krebs NF, Goudar SS, et al. Rates and determinants of early initiation of breastfeeding and exclusive breast feeding at 42 days postnatal in six low and middle-income countries: a prospective cohort study. Reprod Health 2015;12:S10.

26. Berde AS, Yalcin SS. Determinants of early initiation of breastfeeding in Nigeria: a population-based study using the 2013 Demograhic and Health Survey data. BMC Pregnancy Childbirth 2016;16:32.

27. Pérez-Escamilla R, Maulén-Radovan I, Dewey KG. The association between cesarean delivery and breast-feeding outcomes among Mexican women. Am J Public Health 1996;86:832-836.

28. Singh K, Khan SM, Carvajal-Aguirre L, Brodish P, Amouzou A, Moran A. The importance of skin-to-skin contact for early initiation of breastfeeding in Nigeria and Bangladesh. J Glob Health 2017;7:020505.

29. Islam MA, Mamun A, Hossain MM, Bharati P, Saw A, Lestrel PE, et al. Prevalence and factors associated with early initiation of breastfeeding among Bangladeshi mothers: a nationwide crosssectional study. PLoS One 2019;14:e0215733.

30. Ghimire U. The effect of maternal health service utilization in early initiation of breastfeeding among Nepalese mothers. Int Breastfeed J 2019;14:33.

31. Pries AM, Huffman SL, Adhikary I, Upreti SR, Dhungel S, Champeny $\mathrm{M}$, et al. Promotion and prelacteal feeding of breastmilk substitutes among mothers in Kathmandu Valley, Nepal. Matern Child Nutr 2016;12 Suppl 2:8-21.

32. Goldie SJ, Sweet S, Carvalho N, Natchu UC, Hu D. Alternative strategies to reduce maternal mortality in India: a cost-effectiveness analysis. PLoS Med 2010;7:e1000264.

33. Macías YF, Marquis GS, Groleau D, Penny ME. Decline of exclusive breastfeeding: practical advice and stronger policy compliance are needed in government health services in Lima, Peru. FASEB J 2012;26(1 Suppl):806.5.

34. Nkoka O, Ntenda PA, Kanje V, Milanzi EB, Arora A. Determinants of timely initiation of breast milk and exclusive breastfeeding in Malawi: a population-based cross-sectional study. Int Breastfeed J 2019;14:37. 